\title{
Time-scale invariant changes in atmospheric radon concentration and crustal strain prior to a large earthquake
}

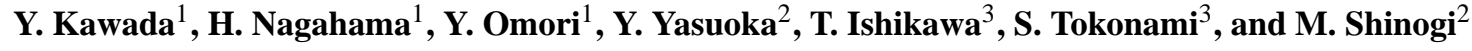 \\ ${ }^{1}$ Department of Geoenvironmental Sciences, Graduate School of Science, Tohoku University, Sendai, Japan \\ ${ }^{2}$ Institute of Radioisotope Research, Kobe Pharmaceutical University, Kobe, Japan \\ ${ }^{3}$ National Institute of Radiological Sciences, Chiba, Japan
}

Received: 21 December 2006 - Revised: 21 February 2007 - Accepted: 5 March 2007 - Published: 19 March 2007

\begin{abstract}
Prior to large earthquakes (e.g. 1995 Kobe earthquake, Japan), an increase in the atmospheric radon concentration is observed, and this increase in the rate follows a power-law of the time-to-earthquake (time-to-failure). This phenomenon corresponds to the increase in the radon migration in crust and the exhalation into atmosphere. An irreversible thermodynamic model including time-scale invariance clarifies that the increases in the pressure of the advecting radon and permeability (hydraulic conductivity) in the crustal rocks are caused by the temporal changes in the power-law of the crustal strain (or cumulative Benioff strain), which is associated with damage evolution such as microcracking or changing porosity. As the result, the radon flux and the atmospheric radon concentration can show a temporal power-law increase. The concentration of atmospheric radon can be used as a proxy for the seismic precursory processes associated with crustal dynamics.
\end{abstract}

\section{Introduction}

Many precursory geochemical anomalies that occurred prior to the 1995 Kobe earthquake in Japan (moment magnitude $M_{w}$ 6.9), were investigated (e.g. Igarashi et al., 1995; Tsunogai and Wakita, 1995, 1996; Johansen et al., 1996, 2000; Yasuoka and Shinogi, 1997; Yasuoka et al., 2006). In particular, Yasuoka and Shinogi (1997) reported an anomaly related to the atmospheric radon $\left({ }^{222} \mathrm{Rn}\right)$ concentration. The concentrations were analyzed statistically and changes were found to follow a power-law of time-to-mainshock (time-to-failure) (Yasuoka et al., 2006). This means that the increase in rate of the atmospheric radon concentration is time-scale invariant, viz., it has a temporal fractal property (e.g. Takayasu, 1990). In general, atmospheric radon is supplied from the

Correspondence to: Y. Kawada

(y-kawada@mail.tains.tohoku.ac.jp) subsurface, and its concentration depends on the meteorological factors such as precipitation and the vertical and horizontal variations in barometric pressure and temperature (e.g. Hatuda, 1953; Ball et al., 1991; Nazaroff, 1992). However, changes in atmospheric radon concentration before a large seismic event was found to be beyond the estimated ranges of annual or daily changes due to meteorological factors (Yasuoka and Shinogi, 1997; Yasuoka et al., 2006). This suggests that anomalous radon exhalation from crust must have occurred prior to the large earthquake.

Holub and Brady (1981) and Katoh et al. (1985) showed an increase in radon emanation associated with microcracking and changes in volumetric strain from uniaxial experiments. Trique et al. (1999) reported that such emanation is associated with transient crustal deformation based on observations of a tunnel in the vicinity of the two lakes. The crustal response due to stress-strain changes in a region prior to large earthquakes is qualified on the basis of cumulative Benioff strain-release (a summation of square root of released energy from each seismic event) following the power-law of time-to-mainshock (time-to-failure). This change has been investigated in terms of the irreversible thermodynamic models such as fibre-bundle and continuum damage models (e.g. Bufe and Varnes, 1993; Bowman et al., 1998; Main, 1999; Rundle et al., 2000; Ben-Zion and Lyakhovsky, 2002; Turcotte et al., 2003; Kawada and Nagahama, 2006). However, the interaction between the crustal response and changes in atmospheric radon concentration has not been studied in terms of the irreversible thermodynamic models and timescale invariance.

In this paper, we investigate the role of crustal deformation in supplying the radon to the atmosphere, in order to explain the cause of increase in atmospheric radon concentration prior to the seismic event. First, we look at the anomalous change in atmospheric radon concentration preceding the 1995 Kobe earthquake, and describe the fundamental principles related to radon migration in the crust. We then

Published by Copernicus GmbH on behalf of the European Geosciences Union and the American Geophysical Union. 


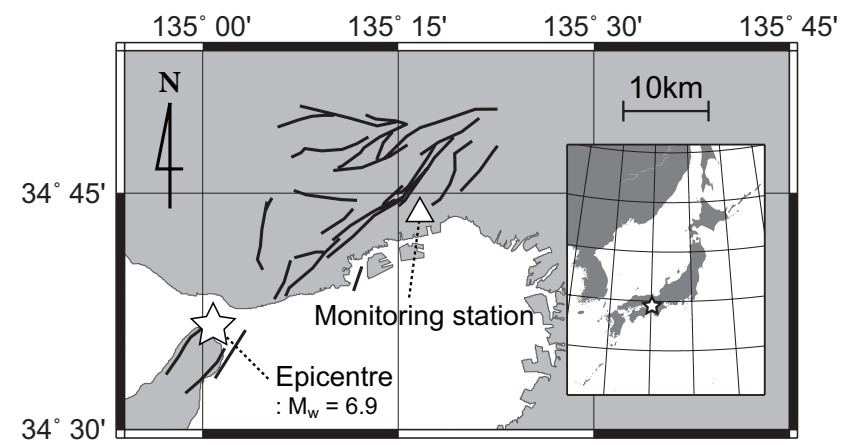

Fig. 1. Map showing the locations of the monitoring station of atmospheric radon concentration, fault distribution in Kobe area (Rokko fault zone) and the epicentre of the 1995 Kobe earthquake (Mizuno et al., 1990; Huzita and Maeda, 1984; Huzita and Kasama, 1982, 1983; Yasuoka et al., 2006).

investigate how the temporal changes in the crustal strain associated with the damage evolution affects the temporal power-law evolution in the atmospheric radon concentration. In particular, the time evolution of porosity, which is a parameter that regulates the migration of radon, will be discussed based on an irreversible thermodynamic model for damage mechanics with time-scale invariance.

\section{Observations and analyses of atmospheric radon con- centration}

Radon $\left({ }^{222} \mathrm{Rn}\right)$ is a radioactive noble gas, having a half-life of 3.8 days. It is the only gaseous nuclide produced as a result of the decay of radium $\left({ }^{226} \mathrm{Ra}\right)$ in naturally radioactive uranium $\left({ }^{238} \mathrm{U}\right)$ series. ${ }^{222} \mathrm{Rn}$ and its short-lived decay products $\left({ }^{218} \mathrm{Po},{ }^{214} \mathrm{~Pb},{ }^{214} \mathrm{Bi}\right.$ and $\left.{ }^{214} \mathrm{Po}\right)$ emit $\alpha$-particles, $\beta$-particles, besides $\gamma$-rays. We can detect the concentration of ${ }^{222} \mathrm{Rn}$ by counting $\alpha$-particle emission, $\beta$-particles and also the $\gamma$-rays (e.g. Ball et al., 1991; Nazaroff, 1992).

We briefly introduce an observation and analysis of atmospheric radon concentration preceding the 1995 Kobe earthquake based on Yasuoka and Shinogi (1997) and Yasuoka et al. (2006). The atmospheric radon concentration was monitored continuously using a flow-type ionization chamber (18 litre volume) from January 1984 to February 1996 (except during January 1989 to December 1989 when the chamber was out of order). The monitoring station is located at the Kobe Pharmaceutical University and directly above the Rokko fault zone (Fig. 1) in which the aftershocks of the Kobe earthquake happen. The air $5 \mathrm{~m}$ above the surface was filtered into the chamber, and the atmospheric radon concentration was measured. The radon decay products are assumed to be trapped by the high-efficiency particulate air filter before measuring the concentration of radon in the ionization chamber.

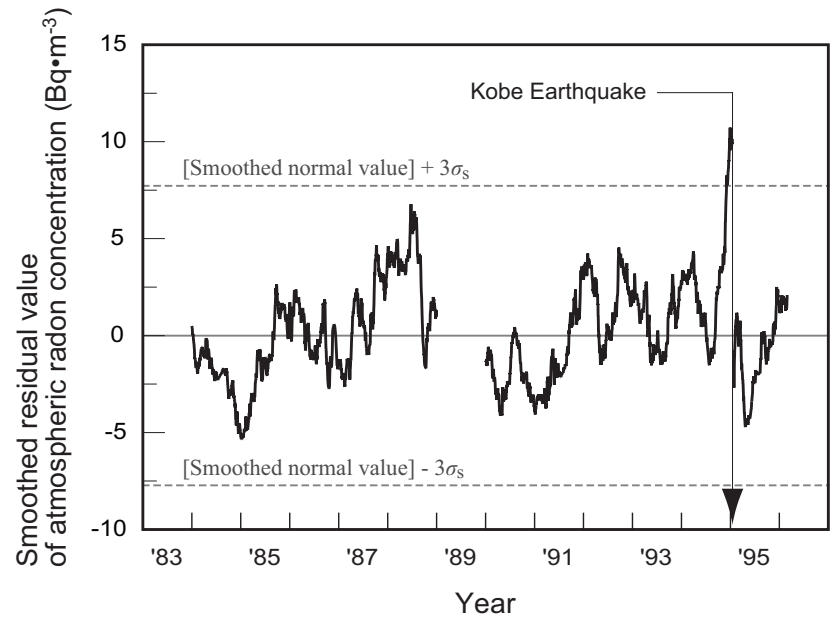

Fig. 2. Time series of the smoothed residual value of atmospheric radon concentration as observed from January 1984 to February 1996 (except from January-December 1989). Data was taken from Yasuoka et al. (2006).

The continuous data was integrated for every hour. The daily minimum in the hourly data was taken to be the regional representative value, since radon is diffused or advected in the atmosphere (Ikebe et al., 1983). The averaged seasonal variation in the daily minimum is taken as the normal value for each day. For example, the normal value for 1 January was derived as the average of nine daily minimums for 1 January observed between 1984 and 1993 (except for 1989 when the data was not available). The daily minimums and normal values of atmospheric radon concentration were smoothed based on the exponential smoothing method (Gardner, 1985) which minimizes daily meteorological effects. Thus, we can quantify the deviation removing the residual seasonal variations in radon concentration as the residual value, i.e., the difference between the smoothed daily minimum and normal value of the radon concentration for each day. The residual values from 1 January 1990 to 30 December 1994 and from 31 December 1994 to 16 January 1995 are smoothed again, respectively, to minimize the meteorological noises more accurately. Thus, we obtain a time-series of the smoothed residual values of atmospheric radon concentration for each day as shown in Fig. 2. Preceding the 1995 Kobe earthquake, a remarkable anomaly was detected - the atmospheric radon concentration was found to continuously exceed the mean by three standard deviations $\left(3 \sigma_{s}\right)$.

To evaluate the seismic precursory anomaly, Yasuoka et al. (2006) analyzed the relation between the time-toearthquake and the smoothed residual values, and showed that the increase in the atmospheric radon concentration $C_{R}$ obeys a power-law of the time to the 1995 Kobe earthquake of the form:

$C_{R}=C_{1}-C_{2}\left(t_{e}-t\right)^{\gamma_{C}}$ 
where $t_{e}$ is the time of occurrence of the 1995 Kobe earthquake, $\gamma_{C}$ is the power-law exponent, and $C_{1}$ and $C_{2}$ are constants (shown in Fig. 3). Equation (1) and Fig. 3 show that the increase in the rate of atmospheric radon concentration is scale invariant during the time-to-failure (timeto-earthquake), i.e., it has a temporal fractal property (e.g. Takayasu, 1990).

The atmospheric radon concentration $C_{R}$ varies linearly with the crustal radon flux, and various regression equations have been proposed (e.g. Ito et al., 1998; Jha et al., 2000; Zhuo et al., 2005). Near the measuring site at the Kobe Pharmaceutical University, the radon flux under normal conditions was estimated to be of the order of $10^{-2} \mathrm{~Bq} \mathrm{~m}^{-2} \mathrm{~s}^{-1}$, and the flux under seismic precursory conditions was found to be 1.9-3.5 times higher than that under normal conditions. These observations and analyses of the anomalous changes in atmospheric radon concentration and radon flux show that the seismic precursory anomaly was mainly attributed to the change in the exhalation of radon from the crust.

\section{Radon migration in the crust and flux to the atmo- sphere}

Uranium $\left({ }^{238} \mathrm{U}\right)$ is distributed widely in crustal rocks so that radon, a nuclide of the natural radioactive ${ }^{238} \mathrm{U}$ series, is constantly generated within the rock strata (e.g. Ball et al., 1991). Radon migrates through cracks in the rock, pores in soil or wide openings along faults or joints, followed by its exhalation into the atmosphere.

Radon is water-soluble and chemically inert, and so its migration occurs through advection of fluid (such as groundwater or crustal gas) as well as via diffusion. The radon diffusion is formulated as Fick's law,

$J=-D \frac{d c}{d z}$,

where $J$ is the molar flux of radon per unit area and time, $D$ is the diffusion constant for radon gas, $d c / d z$ is the gradient of the radon concentration in the interstitial space of the medium ( $\nabla c$ is utilized in case of 3-dimension where $\nabla$ is the gradient operator), and $z$ increases downward (e.g. Hatuda, 1954; Fleischer, 1980; Etiope and Martinelli, 2002). The $D$ value in soil generally ranges from $10^{-7}-10^{-5} \mathrm{~m}^{2} \mathrm{~s}^{-1}$ (e.g. Nazaroff, 1992). On the other hand, the advection of fluid can be expressed using Darcy's law,

$Q=-\kappa \frac{d p}{d z}$,

where $Q$ is the volume flux of the fluid, $\kappa$ is the hydraulic conductivity, and $d p / d z$ is the pressure gradient in the fluid ( $\nabla p$ is substituted for $d p / d z$ in case of 3-dimension). $\kappa$ is defined as $\tilde{\rho} g K / \eta$, where $\tilde{\rho}$ is the density of the fluid, $g$ is the gravitational acceleration, $K$ is the permeability, and $\eta$ is the viscosity of the fluid (e.g. Nazaroff, 1992; Etiope and

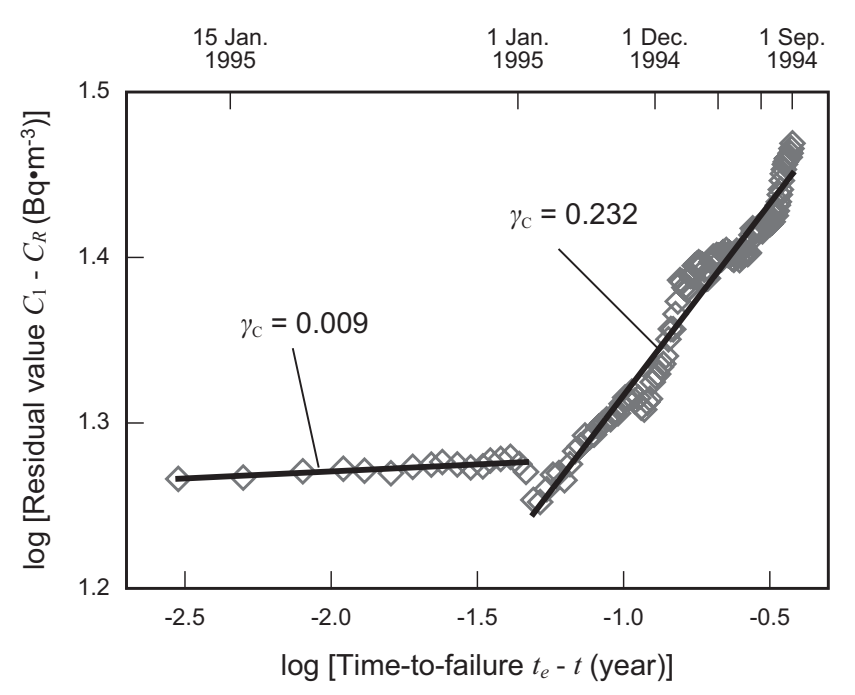

Fig. 3. Log-log plots of the time-to-earthquake (time-to-failure) and smoothed residual value of atmospheric radon concentration during the period from September 1994 to January 1995. The fitted lines follow respective power-laws given in Eq. (1) with $C_{1}=2.86 \times 10$ $\left(\mathrm{Bq} \mathrm{m}^{-3}\right), C_{2}=3.54 \times 10\left(\mathrm{~Bq} \mathrm{~m}^{-3}\right.$ year $\left.^{-\gamma c}\right), t_{e}=1995.045$ (year) and $\gamma_{C}=0.232$, and $C_{1}=2.86 \times 10\left(\mathrm{~Bq} \mathrm{~m}^{-3}\right), C_{2}=1.94 \times 10\left(\mathrm{~Bq} \mathrm{~m}^{-3}\right.$ year $^{-\gamma c}$ ), $t_{e}=1995.045$ (year) and $\gamma_{C}=0.009$. The boundary of the two fitting lines is at 30 December, 1994. Data was obtained from Yasuoka et al. (2006).

Martinelli, 2002). Note that, only the parameter $K$ reflects the rock property. $\kappa$-value ranges in the order of $10^{-11}$ $10^{-4} \mathrm{~m} \mathrm{~s}^{-1}$ for soils (e.g. Bear, 1972) and $10^{-12}-10^{-8} \mathrm{~m} \mathrm{~s}^{-1}$ for limestones and sandstones (e.g. Boving and Grathwohl, 2001). In the Nojima fault, where the 1995 Kobe earthquake occurred, $\kappa$-value was estimated to be of the order of $10^{-6} \mathrm{~m} \mathrm{~s}^{-1}$ from water injection experiments (e.g. Kitagawa et al., 2002), and the $K$-value ranged from $10^{-14}-10^{-13} \mathrm{~m}^{2}$ in the fault breccia zone and $10^{-22}-10^{-18} \mathrm{~m}^{2}$ in the fault gauge, respectively, at effective pressures between 50 and $180 \mathrm{MPa}$ (e.g. Mizoguchi et al., 2000). The increase in the fluxes of $J$ and $Q$ directly reflects the exhalation of radon from crust to atmosphere.

When we neglect the geochemical (such as the radioactive decay rate or spatial distribution of radon) and meteorological (such as barometric pressure) effects of the medium in Eqs. (2) and (3), then $J$ and $Q$ can be seen to depend mainly on the porosity, i.e., a parameter describing number of radon paths in the medium. This is supported by some observations; notably high concentrations of atmospheric radon were detected along many faults (e.g. Ball et al., 1991; Etiope and Martinelli, 2002). This means that the migration of radon depends on the porosity, viz., the volume fraction of interstitial spaces including pores, cracks and the wide openings along the faults. Note that the definition of the term 'porosity' is in a wider sense in this paper. 
The change in porosity is generally affected by the deformation of the medium, i.e., the crustal strain. Holub and Brady (1981) conducted a uniaxial compression experiment with a uranium-bearing granite sample, and showed that temporal change in radon emanation was associated with microcracking of the sample. Moreover, Katoh et al. (1985) also carried out a similar experiment, and pointed out that the increase in volumetric strain is correlated with the radon emanation prior to the failure and that connecting and networking the generated cracks cause an increase in the radon emanation. These results support the fact that the change in crustal strain leads to a corresponding change in atmospheric radon concentration.

\section{Changes in the crustal strain}

Below, we review temporal changes in the crustal strain prior to large earthquakes. Many of the large earthquakes are preceded by an increase in seismic activity in a region called accelerated seismic release (or accelerated moment release) (e.g. Bufe and Varnes, 1993; Bowman et al., 1998; Main, 1999; Rundle et al., 2000; Ben-Zion and Lyakhovsky, 2002; Turcote et al., 2003; Kawada and Nagahama, 2006). This process is defined by cumulative Benioff strain $\Omega$ or cumulative seismic moment $M$ in a region, as follows;

$\Omega=\sum_{k} \sqrt{e_{k}}=\Omega_{c}-A_{\Omega}\left(t_{c}-t\right)^{\gamma_{\Omega}}$,

or

$M=\sum_{k} m_{k}=M_{c}-A_{M}\left(t_{c}-t\right)^{\gamma_{M}}$,

where $e_{k}$ and $m_{k}$ are the energy released and the seismic moment for the $k$-th earthquake, $t_{c}$ is the occurrence time of the large earthquake, $\gamma_{\Omega}$ and $\gamma_{M}$ are exponents of the powerlaw, and $\Omega_{c}, M_{c}, A_{\Omega}$ and $A_{M}$ are constants. Bowman et al. (1998) analyzed the $\gamma_{\Omega}$ value for twelve earthquakes. Rundle et al. (2000) have estimated $\gamma_{\Omega}$ to be $0.26 \pm 0.15$. Note that the Benioff strain is not equal to the crustal strain, while the crustal strain (obtained from satellite data) corresponds to Kostrov strain (which is the cumulative seismic moment $M$ divided by the seismic volume) (Kostrov 1974; Ward, 1998; Main, 1999). These seismo-statistical Eqs. (4) and (5) show a tendency for increase of crustal strain prior to the seismic event, following a power-law time-to-failure equation. Note that the mathematical structure of Eqs. (4) and (5) is same to that of Eq. (1), and the scale invariance of time-to-failure (time-to-earthquake) can be recognized. Moreover, it is known that the scale invariance of time-tofailure also characterizes the ion concentration in groundwater (Johansen et al., 1996, 2000) and also the cumulative Benioff electromagnetic radiation (the square root of the released electromagnetic energy at each earthquake) (e.g. Rabinovitch et al., 2002). Especially, the invariances in cumulative Benioff strain-release and electromagnetic radiation are found to be related to the fractal structure of the pre-existing fault system (Nanjo and Nagahama, 2004) or asperities (e.g. Muto et al., 2007).

Thus, the temporal change in the power-law of crustal (Benioff) strain is associated with the time evolution of porosity and affects the temporal evolution of power-law of the radon exhalation prior to large earthquakes.

\section{Irreversible thermodynamics of radon migration un- der seismic precursory conditions}

\subsection{Damage and porosity evolutions in rocks}

In this section, we consider the time evolution of porosity based on an irreversible thermodynamic model for damage mechanics (e.g. Biot, 1954; Lyakhovsky et al., 1993, 1997; Ben-Zion and Lyakhovsky, 2002; Turcotte et al., 2003; Hamiel et al., 2004; Nanjo and Turcotte, 2005; Kawada and Nagahama, 2006; Kawada et al., 2006). The irreversible thermodynamic model is used to describe the macroscopic viscoelastic (or elastic) behaviour with microscopic damage evolution such as microcracking. The continuum damage model (Lyakhovsky et al., 1993, 1997; Ben-Zion and Lyakhovsky, 2002; Turcotte et al., 2003) and fibre-bundle model (Turcotte et al., 2003; Nanjo and Turcotte, 2005; Kawada and Nagahama, 2006) are different types of the irreversible thermodynamic models.

The damage is quantified by a state parameter $\alpha$, called the damage parameter and lies in the range $0<\alpha<1$. At $\alpha=0$, the material is undamaged, and failed at a critical value of $\alpha_{c}$. Hamiel et al. (2004) discussed the deformation of porous rocks by using this damage parameter and the porosity separately. We consider the two parameters as one, and define the porosity to be the volume fraction of interstitial spaces including cracks, pores and their assemblies along wide fissures such as fault zone, without loss of generality as far as irreversible thermodynamics (e.g. Biot, 1954; Kawada et al., 2006) is concerned. The temporal change in porosity can be linked to damage evolution.

The viscoelastic behaviour of rocks with or without microfracturing is given by Dorn's equation $\dot{\varepsilon} \propto \sigma^{\rho}$ (e.g. Lankford, 1981; Poirier, 1985; Nanjo and Turcotte, 2005). It can be transformed into a temporal power-law in the relaxation modulus $E(t)$, as follows;

$\frac{d \sigma}{d \varepsilon} \equiv E(t) \propto t^{-1 / \rho}$

where $\sigma$ and $\varepsilon$ are the macroscopic elastic stress and strain, respectively. This equation means that the viscoelastic behaviour of rocks with or without microfracturing have an intrinsic invariant time-scale (e.g. Kawada and Nagahama, 2004, 2006; Kawada et al., 2006). Moreover, Schapery $(1964,1969)$ and Kawada et al. (2006) derived the 
stress-strain relation associated with damage evolution based on irreversible thermodynamics as

$\sigma=h_{e} \frac{d \alpha}{d \varepsilon} \int E\left(t-t^{\prime}\right) \frac{d \varepsilon}{d t^{\prime}} d t^{\prime}$,

where $t^{\prime}$ is the arbitrary deformation time, and $h_{e}$, a constant. Note that $\alpha$ contributes towards the plastic component of the deformation. We use Eq. (7) along with Eq. (6) to denote the constitutive law of viscoelastic behaviour with damage evolution.

In this setting, the continuum damage and fibre-bundle models recover the microscopic damage evolution $d \alpha / d t$,

$\frac{d \alpha}{d t} \propto\left(t_{c}-t\right)^{\frac{2-\rho}{\rho-1}}$

and the cumulative Benioff strain-release can be formulated as

$\Omega=\Omega_{c}^{\prime}-B\left(t_{c}-t\right)^{\frac{\rho-2}{2(\rho-1)}}$,

where $\Omega_{c}^{\prime}$ and $B$ are constants (e.g. Lyakhovsky et al., 1993, 1997; Ben-Zion and Lyakhovsky, 2002; Turcotte et al., 2003; Nanjo and Turcotte, 2005; Kawada and Nagahama, 2006). This equation corresponds to Eq. (4), and the exponents are related by $\gamma_{\Omega}=(\rho-2) /[2(\rho-1)]$. Damage evolution given by Eq. (8) has been also investigated based on the percolation model (e.g. Chelidze, 1993; Xie, 1993; Main, 1999; Chelidze et al., 2006).

5.2 Irreversible thermodynamics for radon migration associated with damage evolution

Based on the Sect. 5.1, let us consider damage mechanics with radon migration, defined by Helmholtz free energy of unit volume $F=F(T, \varepsilon, \alpha, c, \zeta)$ where $\varepsilon$ is the macroscopic elastic strain, and $\zeta$ is the volume of the fluid content (e.g. de Groot and Mazur, 1962; Jou et al., 2001; Hamiel et al., 2004). Then, the total differential of $F$ is given by

$d F=T d s+\frac{\partial F}{\partial \varepsilon} d \varepsilon+\frac{\partial F}{\partial \alpha} d \alpha+\frac{\partial F}{\partial c} d c+\frac{\partial F}{\partial \zeta} d \zeta$,

where $s$ is the entropy density, $\partial F / \partial \varepsilon$ can be considered as the macroscopic elastic stress $\sigma, \partial F / \partial c$ as the chemical potential $\mu$, and $\partial F / \partial \zeta$ as the pressure in the fluid $p$. The mass balance equations for radon concentration and volume fluid content are

$\frac{d c}{d t}=-\nabla \cdot J$

$\frac{d \zeta}{d t}=-\nabla \cdot Q$

respectively, where - corresponds to the scalar product. The local entropy production $\Phi$ can be expressed as

$\Phi=\frac{1}{T}\left[\sigma \frac{d \varepsilon}{d t}-\frac{\partial F}{\partial \alpha} \frac{d \alpha}{d t}-J \cdot \nabla \mu-Q \cdot \nabla p\right]$.
When the process is isothermal and linear irreversible, the phenomenological relations for $\alpha, J$ and $Q$ can be defined as

$\frac{d \alpha}{d t}=-\Gamma \frac{\partial F}{\partial \alpha}$

$J=-D^{\prime} \nabla \mu$

$Q=-\kappa^{\prime} \nabla p$,

where $\Gamma$ is a positive constant reflecting the temporal scale of the irreversible process, $D^{\prime} \equiv(\partial c / \partial \mu) D^{\prime \prime}$, where $D^{\prime \prime}$ and $\kappa^{\prime}$ denote a diffusion constant and hydraulic conductivity, respectively. Equation (14) relates to the damage evolution (e.g. Lyakhovsky et al., 1993, 1997; Hamiel et al., 2004; Kawada and Nagahama, 2006; Muto et al., 2007), and Eqs. (15) and (16) recover Eqs. (2) and (3) in one-dimension, respectively (e.g. de Groot and Mazur, 1962; Jou et al., 2001; Hamiel et al., 2004).

We can define Maxwell's relations between each term in Eq. (10). These relations and the mass balance Eqs. (11) and (12) correspond to the field equations in terms of continuum mechanics of porous media (e.g. Biot, 1941, 1956; Rice, 1976). Correspondingly, two Maxwell's relations for the last three terms in Eq. (10) can be expressed as

$\left(\frac{\partial H}{\partial c}\right)_{\varepsilon, \alpha, \zeta}=\left(\frac{\partial \mu}{\partial \alpha}\right)_{\varepsilon, c, \zeta},\left(\frac{\partial H}{\partial \zeta}\right)_{\varepsilon, \alpha, c}=\left(\frac{\partial p}{\partial \alpha}\right)_{\varepsilon, c, \zeta}$,

where $H \equiv \partial F / \partial \alpha$, and the changes in $\mu$ and $p$ are given as

$\delta \mu=\left(\frac{\partial H}{\partial c}\right) \delta \alpha, \delta p=\left(\frac{\partial H}{\partial \zeta}\right) \delta \alpha$,

wherein $\delta$ represents the incremental change. Here, $d \alpha / d t$ in Eq. (8) is defined as a separable function,

$\frac{d \alpha}{d t} \propto G(x, y, z)\left(t_{c}-t\right)^{\frac{2-\rho}{\rho-1}}$,

where function $G(x, y, z)$ relates to the coordinates $(x, y, z)$. Combining Eqs. (15), (16), (18) and (19) leads to

$J=J_{1}-J_{2}\left(t_{c}-t\right)^{\frac{1}{\rho-1}}$,

$Q=Q_{1}-Q_{2}\left(t_{c}-t\right)^{\frac{1}{\rho-1}}$,

where $J_{1}, J_{2}, Q_{1}$ and $Q_{2}$ are constants. From Eqs. (15), (16), (20) and (21), $J$ and $Q$ can be seen to depend on the power-law of time-to-failure (time-to-earthquake). Thus, the cumulative Benioff strain-release is associated with damage evolution, which affects the temporal power-law of exhalation radon from the crust to atmosphere. 


\section{Discussions and conclusions}

We reconsider the migration process of radon under normal condition regulated by Eqs. (2) and (3) and under seismic precursory condition as prescribed by both Eqs. (15) and (16). Comparing the parameters in Eqs. (2), (3), (15) and (16) which decide $J$ and $Q$, under both normal as well as seismic precursory conditions, $D$ and $D^{\prime \prime}$ are found to be nearly equal, whereas, other parameters $\nabla \mu$ (or $\nabla c$ ), $\kappa$ and $\nabla p$ were found to increase under the latter. The damage evolution induces change in the connectivity in the medium (e.g. Chelidze, 1993; Xie, 1993; Chelidze et al., 2006) besides increasing the hydraulic conductivity $\kappa^{\prime}$. Thus, from Eqs. (17) and (18) it can be seen that the damage evolution is associated with changes in parameters $\nabla \mu$ (or $\nabla c), \kappa$ and $\nabla p$, and consequently to the increase in $J$ and $Q$ (or radon flux).

When we regard Eqs. (20) and (21) as Eq. (1), these exponents are related by $\gamma_{C}=1 /(\rho-1)$. In Fig. 3, $\gamma_{C}$-value during September to December 1994 is seen to be equal to 0.232. Hence, $\rho \approx 5.31$, and the exponent of the accelerated seismic release in Eqs. (4) and (9) becomes $\gamma_{\Omega}=0.384$. This $\gamma_{\Omega^{-}}$ value is within the expected value for accelerated seismic release $(0.26 \pm 0.15$, cf. Sect. 3). Although an accelerated seismic release was not observed in the case of the 1995 Kobe earthquake, the atmospheric radon concentration could portray the seismic precursory process associated with crustal dynamics better compared to the small earthquakes that occurred in the region.

In Fig. 3, the regression line can be seen to have a bend at $\left(t_{e}-t\right) \approx 10^{-1.32}$ years, which tells us that the rate of change of atmospheric radon concentration slows down about 15 days prior to the 1995 Kobe earthquake. So, we can infer that the tectonic damage process underwent changes during this period. Log-log plot of the time-series of the atmospheric radon concentration shows the time of this bending clearly. Thus, when there is a detectable anomalous increase in the atmospheric radon concentration which allows us to determine the time when the bending takes place, it is possible to predict the time of mainshock.

There are many modes for radon transportation by fluids such as gas flow in a dry fracture and microbubbles in the groundwater (e.g. Etiope and Martinelli, 2002). By taking into account the rate and distance of the radon migration, the advection of fluids may be the dominant mode. We need to quantify the contribution of each form of radon transportation, but the geochemical and hydrological properties of migration are outside the scope of this paper. In this paper, we shed light into the geophysical or geomechanical properties of the medium through which radon passes, and point out the qualitative relationship between time-scale invariances of the increase in atmospheric radon concentration and damage evolution of the medium.

In conclusion, we have investigated seismic precursory increase in atmospheric radon concentration based on irreversible thermodynamics for the damage mechanics of the crust supplying the radon to atmosphere. The temporal power-law change in the cumulative Benioff strain was found to be associated with the damage evolution viz., the change in porosity. This time-scale invariant behaviour of the crust promotes an increase in the hydraulic conductivity (permeability), pressure of the fluid advecting radon, and the radon concentration within the crustal rocks, all of which affect the time-scale invariant increase in the flux of radon into the atmosphere and hence the atmospheric radon concentration. The concentration of atmospheric radon can be used as a proxy for the seismic precursory processes associated with crustal dynamics.

Acknowledgements. The authors thank V. Lyakhovsky, A. Tarquis and an anonymous reviewer for critical reviews and meaningful comments, H. Sato and K. Otsuki for insightful comments, T. Yajima for valuable comments related to the mathematical procedure of irreversible thermodynamics, and D. Nair for revising the English style of this manuscript. This study was conducted as a part of the 21st Century Center-Of-Excellence program, "Advanced Science and Technology Center for the Dynamic Earth", of Tohoku University. The first author (Y. Kawada) is financially supported by the JSPS Research Fellowship for Young Scientists.

Edited by: A. Tarquis

Reviewed by: V. Lyakhovsky and another referee

\section{References}

Ball, T. K., Cameron, D. G., Colman, T. B., and Roberts, P. D.: Behaviour of radon in the geological environment: a review, Quart. J. Engng. Geol., 24, 169-182, 1991.

Bear, J.: Dynamics of Fluids in Porous Media, Elsevier, New York, 1972.

Ben-Zion, Y. and Lyakhovsky, V.: Accelerated seismic release and related aspects of seismicity patterns on earthquake faults, Pure Appl. Geophys., 159, 2385-2412, 2002.

Biot, M. A.: General theory of three-dimensional consolidation, J. Appl. Phys., 12, 155-164, 1941.

Biot, M. A.: Theory of stress-strain relation in anisotropic viscoelasticity and relaxation phenomena, J. Appl. Phys., 25, 13851391, 1954.

Biot, M. A.: General solutions of the equations of elasticity and consolidation for a porous material, Trans. ASME, J. Appl. Mech., 23, 91-96, 1956.

Boving, T. B. and Grathwohl, P.: Tracer diffusion coefficients in sedimentary rocks: correlation to porosity and hydraulic conductivity, J. Contam. Hydrol., 53, 85-100, 2001.

Bowman, D. D., Ouillon, G., Sammis, C. G., Sornette, A., and Sornette, D.: An observational test of the critical earthquake concept, J. Geophys. Res., 103B, 24 359-24 372, 1998.

Bufe, C. G. and Varnes, D. J.: Predictive modeling of the seismic cycle of the Greater San Francisco Bay region, J. Geophys. Res., 98B, 9871-9883, 1993.

Chelidze, T.: Fractal damage mechanics of geomaterials, Terra Nova, 5, 421-437, 1993. 
Chelidze, T., Kolesnikov, Yu., and Matcharashvili, T.: Seismological criticality concept and percolation model of fracture, Geophys. J. Int., 164, 125-136, 2006.

de Groot, S. R. and Mazur, P.: Non-equilibrium Thermodynamics, North-Holland, Amsterdam, 1962.

Etiope, G. and Martinelli, G.: Migration of carrier and trace gases in the geosphere: an overview, Phys. Earth Planet. Int., 129, 185204, 2002.

Fleischer, R. L.: Radon flux from the earth: methods of measurement by the nuclear track technique, J. Geophys. Res., 85C, 7553-7556, 1980.

Gardner, E. S.: Exponential smoothing: The state of art, J. Forcasting, 4, 1-28, 1985.

Hamiel, Y., Lyakhovsky, V., and Agnon, A.: Coupled evolution of damage and porosity in poroelastic media: theory and applications to deformation of porous rocks, Geophys. J. Int., 156, 701$713,2004$.

Hatuda, Z.: Radon content and its change in soil air near the ground surface, Mem. Col. Sci. Univ. Kyoto, Ser. B, 20, 285-306, 1953.

Hatuda, Z.: Radioactive method for geological exploration, Mem. Coll. Sci. Univ. Kyoto, Ser. B, 21, 231-271, 1954.

Holub, R. F. and Brady, B. T.: The effect of stress on radon emanation from rock, J. Geophys. Res., 86B, 1776-1784, 1981.

Huzita, K. and Kasama, T.: Geology of the Osaka-Seihokubu District, Quadrangle Series, with Geological Sheet Map at 1:50 000, Geol. Surv. Japan, Tsukuba, 1982.

Huzita, K. and Kasama, T.: Geology of the Kobe District, Quadrangle Series, with Geological Sheet Map at 1:50 000, Geol. Surv. Japan, Tsukuba, 1983.

Huzita, K. and Maeda, Y.: Geology of the Suma District, Quadrangle Series, with Geological Sheet Map at 1:50000, Geol. Surv. Japan, Tsukuba, 1984.

Igarashi, G., Saeki, S., Takahata, N., Sumikawa, K., Tasaka, S., Sasaki, Y., Takahashi, M., and Sano, Y.: Ground-water radon anomaly before the Kobe earthquake in Japan, Science, 269, 6061, 1995.

Ikebe, Y., Kojima, S., and Shimo, M.: On the origin and transport of Rn-222 in the atmosphere, Res. Lett. Atmos. Electr., 3, 51-54, 1983.

Ito, K., Ishimori, Y., and Furuta, S.: Environmental effects of radon and its progeny from uranium waste rock piles: part 2 - measurements by grab sampling methods, Radon and Thoron in the human environment, Proc. 7th Tohwa Univ. Int. Symp., Fukuoka, Japan, edited by: Katase, A. and Shimo, M., Singapore, World Scientific, 288-293, 1998.

Jha, S., Khan, A. H., and Mishra, U. C.: A study of the ${ }^{222}$ Rn flux from soil in the $\mathrm{U}$ mineralised belt at Jaduguda, J. Environ. Radioact., 49, 157-169, 2000.

Johansen, A., Sornette, D., Wakita, H., Tsunogai, U., Newman, W. I., and Saleur, H.: Discrete scaling in earthquake precursory phenomena: evidence in the Kobe earthquake, Japan, J. Phys. I (France), 6, 1391-1402, 1996.

Johansen, A., Saleur, H., and Sornette, D.: New evidence of earthquake precursory phenomena in the 17 January 1995 Kobe earthquake, Japan, Eur. Phys. J. B, 15, 551-555, 2000.

Jou, D., Casas-Vázquez, J., and Lebon, G.: Extended Irreversible Thermodynamics, 3rd Ed., Springer-Verlag, Berlin, 2001.

Katoh, K., Nishizawa, O., Kuronose, K., and Ikeda, K.: An experimental study on variation of radon emanation from Westerly
Granite under uniaxial compression Part 1, Jisin II. (in Japanese, with English abstr.), 38, 173-182, 1985.

Kawada, Y. and Nagahama, H.: Viscoelastic behaviour and temporal fractal properties of lherzolite and marble: possible extrapolation from experimental results to the geological time-scale, Terra Nova, 16, 128-132, 2004.

Kawada, Y. and Nagahama, H.: Cumultative Benioff strain-release, modified Omori's law and transient behaviour of rocks, Tectonophysics, 424, 157-166, 2006.

Kawada, Y., Nagahama, H., and Hara, H.: Irreversible thermodynamic and viscoelastic model for power-law relaxation and attenuation of rocks, Tectonophysics, 427, 255-263, 2006.

Kitagawa, Y., Fujimori, K., and Koizumi, N.: Temporal change in permeability of the rock estimated from repeated water injection experiments near the Nojima fault in Awaji Island, Japan, Geophys. Res. Lett., 29, 1483, doi:10.1029/2001GL014030, 2002.

Kostrov, V. V.: Seismic moment and energy of earthquakes, and seismic flow of rock, Izv. Acad. Sci. USSR Phys. Solid Earth, 1, 23-40, 1974.

Lankford, J.: The role of tensile microfracture in the strain rate dependence of compressive strength of fine-grained limestone analogy with strong ceramics, Int. J. Rock Mech. Min. Sci. \& Geomech. Abstr., 18, 173-175, 1981.

Lyakhovsky, V., Ben-Zion, Y., and Agnon, A.: Distributed damage, faulting, and friction, J. Geophys. Res., 102B, 27 635-27 649, 1997.

Lyakhovsky, V., Podladchikov, Yu., and Poliakov, A.: A rheological model of a fractured solid, Tectonophysics, 226, 187-198, 1993.

Main, I. G.: Applicability of time-to-failure analysis to accelerated strain before earthquakes and volcanic eruptions, Geophys. J. Int., 139, F1-F6, 1999.

Mizoguchi, K., Hirose, T., and Shimamoto, T.: Permeability structure of the Nojima fault at Funaki, Hokudan-cho, Japan, Gekkanchikyu-gougai (Earth Monthly) (in Japanease), 31, 5865, 2000.

Mizuno, K., Hattori, H., Sangawa. A., and Takahashi, Y.: Geology of the Akashi District, Quadrangle Series, with Geological Sheet Map at 1:50 000, Geol. Surv. Japan, Tsukuba, 1990.

Muto, J., Nagahama, H., Miura, T., and Arakawa, I.: Frictional discharge at fault asperities: Origin of fractal seismoelectromagnetic radiation, Tectonophysics, 431, 113-122, 2007.

Nanjo, K. and Nagahama, H.: Fractal properties of spatial distributions of aftershocks and active faults, Chaos, Solitons \& Fractals, 19, 387-397, 2004.

Nanjo, K. Z. and Turcotte, D. L.: Damage and rheology in a fibrebundle model, Geophys. J. Int., 162, 859-866, 2005.

Nazaroff, W. W.: Radon transport from soil to air, Rev. Geophys., 30, 137-160, 1992.

Poirier, J. P.: Creep of Crystals, Cambridge Univ. Press, New York, 1985.

Rabinovitch, A., Frid, V., and Bahat, D.: GutenbergRichter-type relation for laboratory fracture-induced electromagnetic radiation, Phys. Rev. E, 65, 011401, doi:10.1103/PhysRevE.65.011401, 2002.

Rice, J. R. and Cleary, M. P.: Some basic stress diffusion solutions for fluid-saturated elastic porous media with compressible constituents, Rev. Geophys. Space Phys., 14, 227-241, 1976.

Rundle, J. B., Klein, W., Turcotte, D. L., and Malamud, B. D.: Precursory seismic activation and critical-point phenomena, Pure 
Appl. Geophys., 157, 2165-2182, 2000.

Schapery, R. A.: Application of thermodynamics to thermomechanical, fracture, and birefringent phenomena in viscoelastic media, J. Appl. Phys., 35, 1451-1465, 1964.

Schapery, R. A.: On the characterization of nonlinear viscoelastic materials, Polym. Eng. Sci., 9, 295-310, 1969.

Takayasu, H.: Fractals in the Physical Sciences, Manchester Univ. Press, Manchester, 1990.

Trique, M., Richon, P., Perrier, F., Avouac, J. P., and Sobroux, J. C.: Radon emanation and electric potential variation associated with transient deformation near reservoir lakes, Nature, 399, 137-141, 1999.

Tsunogai, U. and Wakita, H.: Precursory chemical changes in ground water: Kobe earthquake, Japan, Science, 269, 61-63, 1995.

Tsunogai, U. and Wakita, H.: Anormalous changes in groundwater chemistry - possible precursors of the 1995 Hyogo-ken Nanbu earthquake, Japan, J. Phys. Earth, 44, 381-390, 1996.
Turcotte, D. L., Newman, W. I., and Shcherbakov, R.: Micro and macroscopic models of rock fracture, Geophys. J. Int., 152, 718$728,2003$.

Ward, S. N.: On the consistency of earthquake moment rates, geological fault data, and space geodetic strain: the United States, Geophys. J. Int., 134, 172-186, 1998.

Xie, H.: Fractals in Rock Mechanics, Balkema, Rotterdam, 1993.

Yasuoka, Y. and Shinogi, M.: Anomaly in atmospheric radon concentration: a possible precursor of the 1995 Kobe, Japan, earthquake, Health Phys., 72, 759-761, 1997.

Yasuoka, Y., Igarashi, G., Ishikawa, T., Tokonami, S., and Shinogi, M.: Evidence of precursor phenomena in the Kobe earthquake obtained from atmospheric radon concentration, Appl. Geochem., 21, 1064-1072, 2006.

Zhuo, W., Furukawa, M., Guo, Q., and Kim, Y. S.: Soil radon flux and outdoor radon concentrations in East Asia, Int. Congr. Ser., 1276, 285-286, 2005. 